\title{
Evaluation of Proportional and Discrete Shared Control Paradigms for Low Resolution User Inputs
}

\author{
Tom Carlson, Guillaume Monnard, Robert Leeb and José del R. Millán \\ Chair in Non-invasive Brain-Machine Interface (CNBI) \\ École Polytechnique Fédérale de Lausanne (EPFL) \\ Switzerland \\ tom.carlson@epfl.ch
}

\begin{abstract}
For people with severe physical disabilities, low resolution input devices, such as buttons, sip and puff switches and brain-computer interfaces provide an opportunity to interact with the world. However, it can be difficult to control assistive technology, such as wheelchairs, tele-presence robots and robotic arms, when you have only a limited number of commands available and/or a lack of temporal precision in issuing such commands. These limitations can be overcome by employing shared control techniques, whereby the system assists the user in performing the desired task. In this study we compare the use of a simple discrete shared control policy with a more dynamic proportional shared control policy. We evaluate both approaches on a wheelchair that is only operated by two temporallyconstrained discrete buttons. The experiments were performed in two different realistic indoor scenarios: an open-plan, spacious environment and a smaller, more cluttered office environment. A total of $\mathbf{1 0}$ healthy participants took part in this study.

Index Terms-Shared control, HRI, assistive technology, user evaluation
\end{abstract}

\section{INTRODUCTION}

Shared control is a vital component that will empower people to use assistive technology effectively and through it, gain a degree of independence. There have been many studies that report an improvement in some performance metric when a task is attempted using shared control [1], [2]. However there is little literature that compares different shared control strategies, especially with respect to the user performance: a comprehensive review of the types of evaluation methodologies for assistive technology is given in [3].

In particular, shared control has been shown to increase safety and decrease user workload [2], both of which are important for an end user. However, it is also known that people find it difficult to form mental models of systems that dynamically adapt their behaviour in order to provide such assistance [4], [5]. To overcome this difficulty, we believe that the shared control behaviour must be intuitive and that the user must be given appropriate feedback.

In this paper, we begin by discussing the nature of the two shared control policies. We then we describe their implementation on our powered wheelchair platform, which is capable of detecting obstacles in the environment. The protocols that we used for the experiments are defined and the results are analysed. The two approaches are found to differ considerably in a cluttered environment, whereas the overall performance
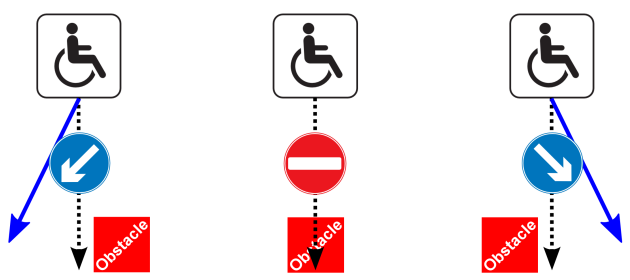

Fig. 1: The principle of shared control for obstacle avoidance.

changed very little in an open-plan environment. Although proportional shared control did not significantly change the performance in easier to handle environments-where it was sufficient to use discrete shared control-we conclude that overall it is advantageous to use proportional shared control, since it enhanced performance in the most difficult situations.

\section{The Shared CONTROLler}

Many approaches exist for sharing control between man and vehicle, ranging from those that offer low-level collision avoidance [6], to those that operate almost autonomously, predicting long-term user goals [7] and even those that use haptics to physically guide users towards giving suitable input signals to the system [8]. In our case, we are interested in assisting the user in the short-term, local environment, allowing the user to asynchronously initiate manoeuvres and maintain the high-level control of the system to achieve their desired goals (Fig. 1). It is important that the user is able to make the most of his or her abilities [9], so the wheelchair should only assist where absolutely necessary. We have taken two approaches to shared control for this experiment: discrete (reactive) and proportional (proactive).

\section{A. Discrete Shared Control (DSC)}

The discrete shared control policy was designed to be deliberately simple in order to facilitate the formation of a mental model of the behaviour of the wheelchair. In related studies it was suggested that adaptive shared control techniques can be difficult for the user to understand [2]. Given that it is not easy for users to transition from one wheelchair to another, simply due to the change in dynamics [10], it is probably best 
to make the shared control module as predictable (simple) as possible.

In the discreet shared control paradigm, three zones were defined in the area in front of the wheelchair. These corresponded to the left, centre and right areas up to $0.55 \mathrm{~m}$ in front of the wheelchair's footplate. If the obstacle densities in these regions surpass an empirically set threshold, the wheelchair will stop. Additionally, when the wheelchair receives a turn command, it will continue to turn until it either reaches a maximum of $45^{\circ}$, or the obstacle density in the turning zone (i.e. left or right zone) surpasses the same threshold.

\section{B. Proportional Shared Control (PSC)}

The assistance from the discrete shared controller is reactive in the sense that any change in direction, however small is user initiated. In the case of low resolution input devices, this puts a potentially unnecessary burden on the user to give many relatively low level commands. However, it has been suggested that some patient groups would benefit from systems that could take a high level command and drive with a little more autonomy. For example, to pro-actively avoid obstacles, dock to desks and prevent zig-zag motions down corridors, thus reducing the unnecessary user workload [11].

In this paradigm, the wheelchair pro-actively slows down and turns to avoid obstacles as it approaches them from a greater distance. We extended our discrete shared control implementation, by using the obstacle densities in the left and right zones to apply virtual forces that affect the rotational velocity of the chair, whereas the obstacle density in the centre front was used to control the translational velocity of the wheelchair, in a similar manner to [12].

\section{System Setup}

The shared controller is dependent on three key pieces of information: the user intent, the location of potential targets that the user may wish to reach and the location of potential obstacles to avoid, as shown in the system diagram (Fig. 2.)

\section{A. User Intent}

Eventually, we intend to infer the user's intention from the output of a non-invasive brain-computer interface (BCI) [13], [14]. The output from the BCI device will asynchronously output a turn left or turn right command, according to the mental task the user is performing. In [1], it was shown that by using a 2-class sensorimotor-rhythm (SMR) based BCI coupled with shared control, similar levels of performance to controlling a device manually with two buttons could be reached. Since the training and setup phases of a BCI can be very timeconsuming [15], in this study, we use a discrete two-button input device to represent the output of the two BCI classes. This allows us to test our system and determine benchmark performance levels of the shared control paradigms, before undertaking a full BCI experiment. By taking this approach, we also avoid frustrating precious BCI participants, by ensuring users can reach a minimum required level of performance, before introducing new parameters (e.g. the uncertainty of the $\mathrm{BCI}$.

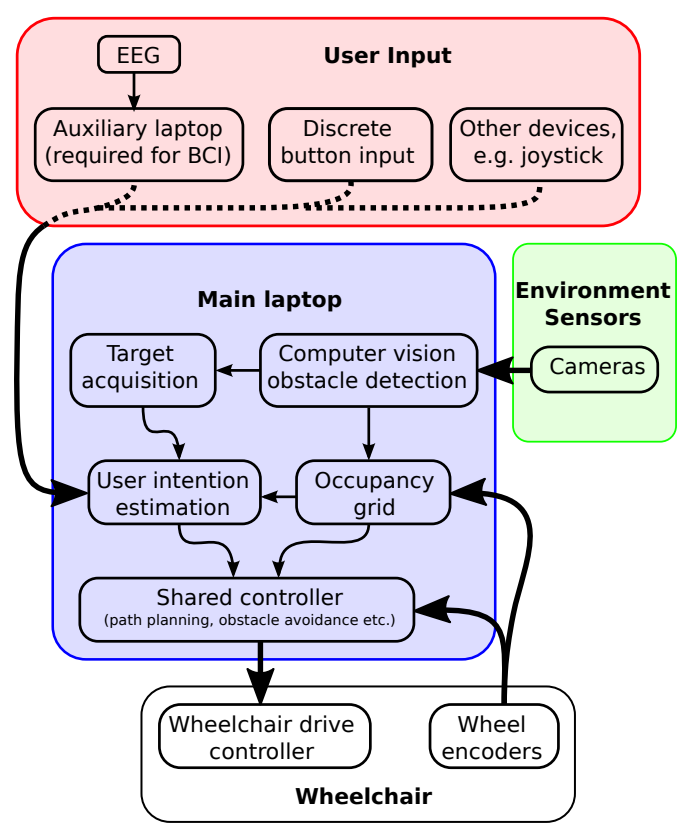

Fig. 2: The wheelchair block diagram. We use the same generic architecture in all conditions in our study; we only change the shared control module or the user input device. The actual user input devices are interchangeable and need only generate a high-level desired direction (e.g. left or right).
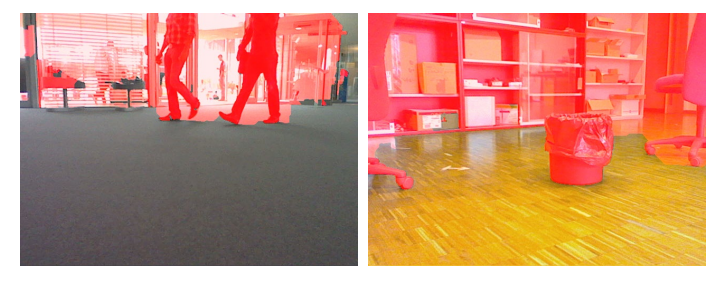

Fig. 3: Examples of detected obstacles being highlighted.

\section{B. Target Acquisition}

For this study, we consider any obstacle to be a potential target, provided it is located directly in front of the wheelchair. This way, the obstacle avoidance routine will only attempt to avoid obstacles that lie slightly to one side or the other of the wheelchair's heading. Consequently, the user is able to dock to (but not hit) any "obstacle", be it a person, table, or even a wall. In future, it may be desirable to acquire potential targets and infer user intentions by using method similar to that proposed in [16], especially if the environment is mapped, or to extrapolate this method for unmapped environments.

\section{Obstacle Detection}

In this study, cameras were the only sensors used to perceive the environment. Using the computer vision algorithm described in [17] (see Fig. 3), we construct a local $10 \mathrm{~cm}$ resolution occupancy grid [18], which is then used by the shared control module for local planning. 


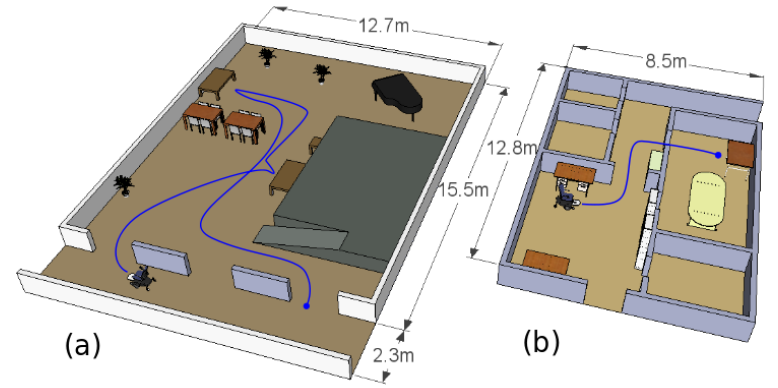

Fig. 4: The task to be performed in (a) the spacious, open-plan environment and (b) the cluttered office environment.

TABLE I: Experimental protocol

(a) 15 minutes familiarisation with the two-button control of the wheelchair (b) $\mathrm{N}$ runs of $\mathrm{X}$ shared control

(c) 1 familiarisation run with Y shared control

(d) $\mathrm{N}$ runs of $\mathrm{Y}$ shared control

NOTE: In the trials, $\mathrm{X}$ and $\mathrm{Y}$ are counterbalanced and represent either the reactive discrete or proactive proportional shared control paradigms. $\mathrm{N}=2$ for the open-plan environment and $\mathrm{N}=3$ for the cluttered environment.

\section{EXPERIMENTAL DESIGN}

Two different scenarios were investigated: driving in an open-plan spacious environment, where the smallest doorway was around twice the width of the wheelchair (Fig. 4(a)) and performing manoeuvres in a cluttered environment, where the smallest doorway was approximately $10 \mathrm{~cm}$ wider than the wheelchair (Fig. 4(b)). In each run of the particular scenario the participant was asked to drive along the route shown in Fig. 4. This involved performing typical everyday manoeuvres, such as passing through doorways, navigating around obstacles, including tables, chairs and ornamental trees as well as docking to tables.

The counterbalanced experimental protocol is summarised in Table I. Four healthy volunteers drove in the open-plan environment and six healthy volunteers performed the manoeuvres in the more cluttered environment. An appropriate description of the wheelchair control paradigm was given to each participant before they began their familiarisation phases (Table I):

- Discrete shared control: "When the wheelchair sees an obstacle, it will highlight it in red on the screen (see Fig. 3) and will stop in front of the obstacle. When you deliver a turn left or right command, the wheelchair will decide how far to turn, depending on the presence or absence of obstacles in the surrounding area."

- Proportional shared control: Additionally, "If the wheelchair is aligned with the obstacle, it will gradually slow down and eventually stop in front of the obstacle. If the obstacle is not in the centre of the wheelchair's path, the wheelchair will adjust it's trajectory to avoid the obstacle."

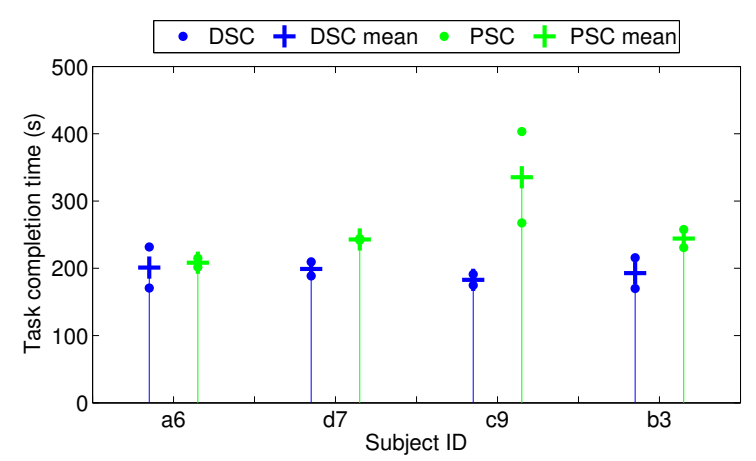

Fig. 5: Task completion time: crosses denote mean values ([Discrete/Propotional] Shared Control) $(p=0.1329)$.

\section{RESULTS}

Although the dataset is relatively small, in the interest of completeness, we investigated the statistical significance of our results using the paired Student's $t$-test. This was done by concatenating the results from each trial for all the subjects to see if the results were significant across the population.

\section{A. The Open-plan Environment}

Four healthy subjects (aged 23-28) participated in this part of the study, which took place in the environment shown in Fig. 4(a). The following metrics were calculated offline to evaluate the system and user performance after the experiments. Overall the performance appeared to be slightly worse, when using proportional shared control, compared with discrete shared control, however none of these differences in performance proved to be statistically significant, as will be discussed.

1) Task Success Rate: To complete the task successfully, the participant had to drive through a doorway, reach two tables and reach the final exit doorway. All participants were able to complete the entire task for every trial under both conditions in the spacious environment. They drove approximately $40 \mathrm{~m}$, with no significant difference between conditions with respect to the total distance covered ( $p=0.2529$ ).

2) Task Completion Time: On average, across all subjects and trials, it took 3'14' to complete the task using discrete shared control and 4'27' when using proportional shared control, although this may be influenced by a potential outlier in one of subject c9's runs (Fig. 5). The difference was not a statistically significant $(p=0.1329)$.

3) Average Wheelchair Speed: There was a corresponding slight decrease in average wheelchair speed from $0.2 \mathrm{~ms}^{-1}$ with discrete shared control to $0.17 \mathrm{~ms}^{-1}$ when using the proportional controller (Fig. 6). Again this difference was not statistically significant $(p=0.0990)$.

4) Number of Stops: The shared controller will stop the wheelchair if a collision is imminent and indeed the wheelchair is required to stop when it reaches the user's intended destination. However, there were two areas in the room that exhibited excessive reflections from the plant pots and triggered a 


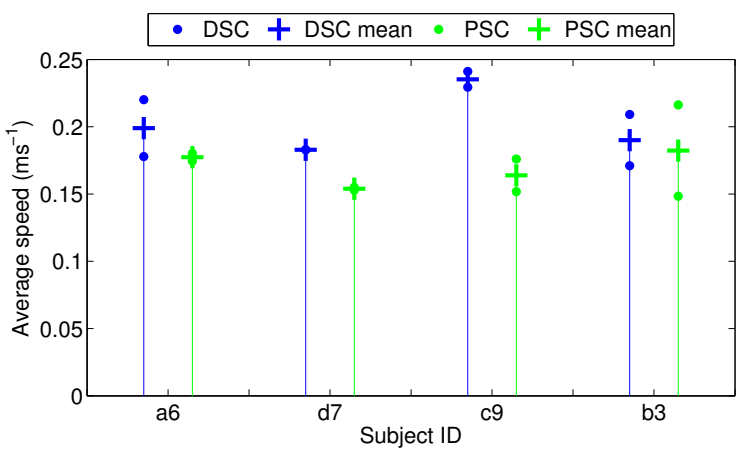

Fig. 6: Average wheelchair speed ([Discrete/Propotional] Shared Control) $(p=0.0990)$.

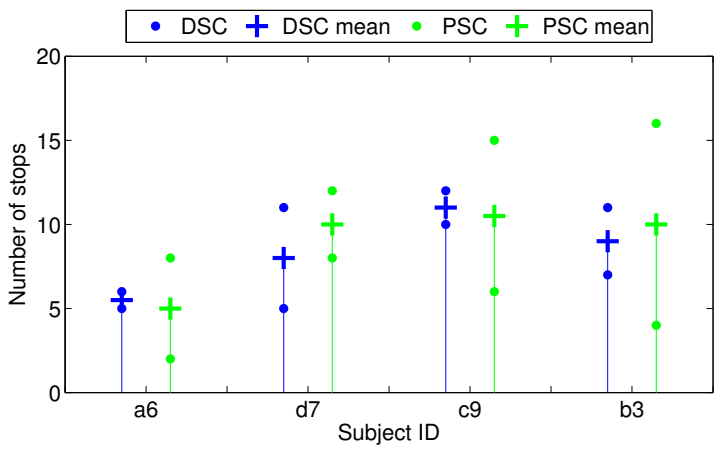

Fig. 7: Number of stops ([Discrete/Propotional] Shared Control) $(p=0.4740)$.

number of false positives in the computer vision based obstacle detection algorithm. These caused the wheelchair to make some additional stops that the participants had to circumvent. Too many unnecessary stops, whether caused by false positives in the obstacle detection, or an imprecise/over-cautious controller, may well frustrate the user. In this environment, there was no significant difference $(p=0.4740)$ in the average number of stops made by either controller (Fig. 7). On average, there were 8 stops per task, including the 3 desired stops (once at each of the two tables and once at the end of the course.

5) Number of Commands: We had designed a balanced experimental course, such that participants would be required to execute approximately the same number of left and right turns. This is confirmed by the results shown in Fig. 8. Again, there was no significant difference between the two conditions.

6) Assistance Factor: The assistance factor, $A F$, gives us an idea of how much the wheelchair's shared controller was contributing to the actual driving of the wheelchair and is defined as:

$$
A F=1-\frac{N_{c}-N_{b}}{N_{c}+N_{p}},
$$

where $N_{c}$ is the total number of commands the user delivered, $N_{b}$ is the number of commands the shared control blocked from being executed and $N_{p}$ is the number of proactive

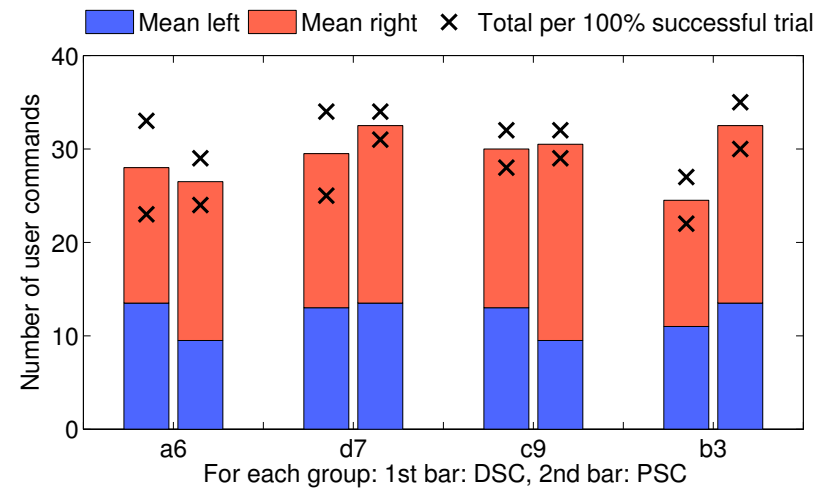

Fig. 8: Number of user commands ([Discrete/Propotional] Shared Control) $(p=0.3101)$.

commands the shared control executed, without any explicit input from the user.

Since the shared controller cannot block more commands than were executed, the $A F$ metric is constrained to lie on the interval $[0,1]$. When $A F=0$, it means the shared controller did not give the user any help to execute the task, at the other extreme, when $A F=1$, the wheelchair completed the task purely autonomously. In practice we never reach the latter, fully autonomous case, although it may be observed over very short, simple trajectories.

The assistance factor was significantly higher in the proportional condition ( 0.67 as opposed to $0.10, p=0.0089$ ), due to the fact that the proportional shared controller was capable of making proactive turns, whereas the discrete shared controller was only reactive, in the sense that it blocked dangerous commands that were delivered by the user, or stopped the wheelchair if a collision was imminent.

7) Qualitative Analysis: After the experiments, each participant was asked if they noticed any difference in the wheelchair's behaviour. None of the participants noticed any difference between the control modes and they did not express a preference towards either driving with either mode.

Additionally, one of the subjects remarked that they felt slightly frustrated by the false positives in the obstacle detection, since it was hard to understand why the wheelchair was stopping on some occasions. Although this is a point that we will strive to address in our future work, we do not believe it affected the comparison between the two control modes, since there was no significant difference in the prevalence of these false positives between conditions (Fig. 7).

\section{B. The Cluttered Environment}

Six healthy subjects (aged 23-26) participated in this part of the study, which was undertaken in the environment shown in Fig. 4(b). When we used the same control paradigms in a cluttered environment-where higher precision driving was required-we obtained a striking difference between the discrete and proportional shared controller in all our metrics. Typical trajectories that were driven are shown in Fig. 9. The 


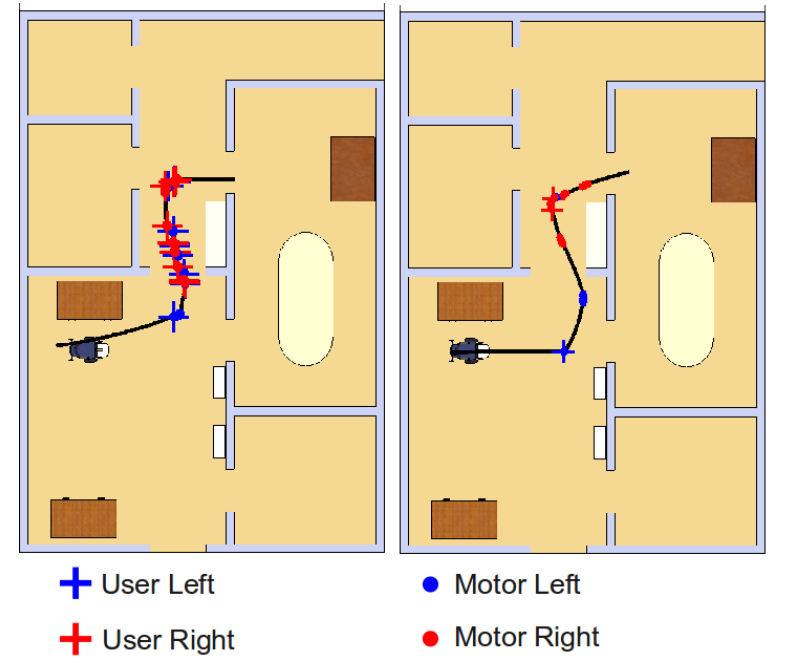

Fig. 9: Commands delivered along typical trajectories that were driven using discrete control (left figure) and proportional control (right figure) in the cluttered environment.

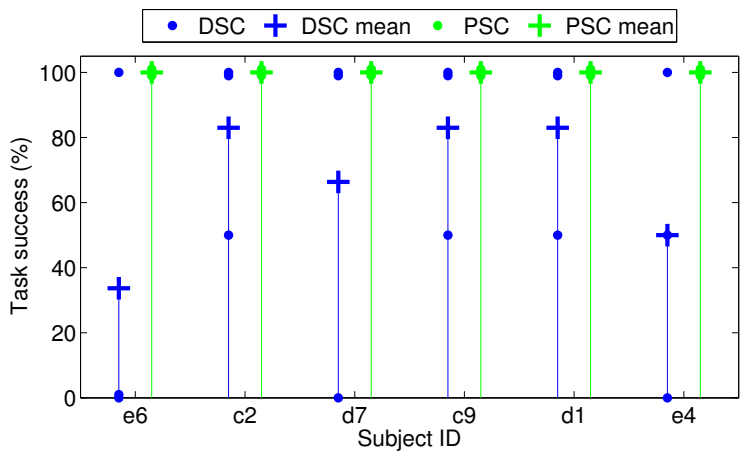

Fig. 10: Task success rate: percent of the task completed succesfully ([Discrete/Propotional] Shared Control) $(p=0.0117)$.

crosses indicate the position of the wheelchair when the subject issued a turning command. Similarly the thicker, blue and red coloured parts of the trajectory show where the controller issued a left or right motor command respectively. If there is not a corresponding cross, the command is a proactive one.

1) Task Success Rate: The task involved navigating through two doorways. In this metric each doorway was equally weighted, so the task completion was simply $50 \%$ per doorway that the participant managed to safely navigate through. When using the proportional shared controller, all trials were successfully completed, however, when the discrete shared controller was used, there was a significant $(p=0.0117)$ drop by a third in the task completion to an average value of $67 \%$.

Note: The remaining metrics were computed for the trials where the task was fully completed.

2) Task Completion Time: Again, there was a very significant $(p=0.0068)$ improvement in task completion time, from an average of 113 seconds with discrete shared control, to only

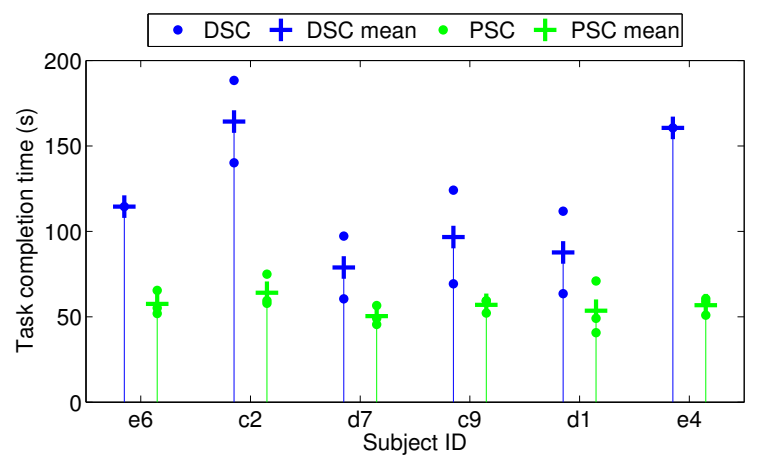

Fig. 11: Task completion time ([Discrete/Propotional] Shared Control) $(p=0.0068)$.

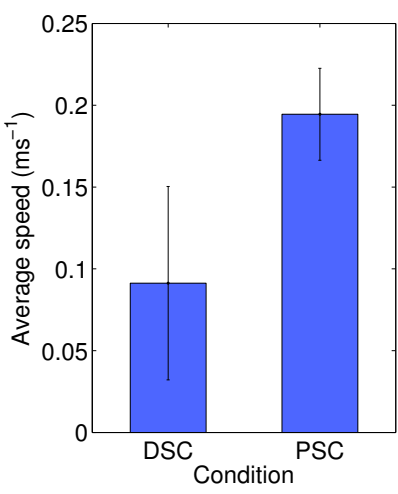

(a)

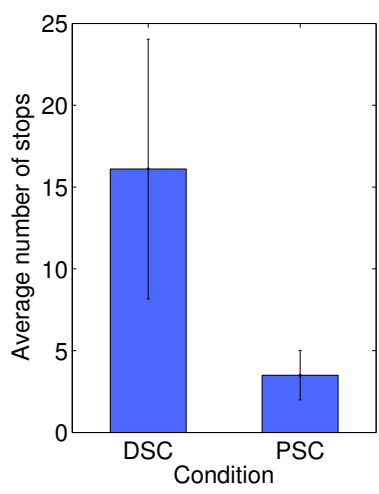

(b)
Fig. 12: (a) Average wheelchair speed $(p=0.0017)$, (b) Average number of stops $(p=0.0037)$ ([Discrete/Propotional] Shared Control)

57 seconds when using proportional shared control. It is also worth noting, in Fig. 11, the reduced inter-subject variance for task completion time, when using the proportional shared controller. This is probably due to the proactive commands that make the task easier and to perform and therefore yields more uniform trajectories.

3) Average Wheelchair Speed: and

4) Number of Stops: We can see from Fig. 12a that the average wheelchair speed is significantly higher $(p=0.0017)$ when using proportional shared control. This is most likely due to the significantly ( $p=0.0037$ ) reduced number of stops the wheelchair made, compared with the discrete shared control condition, as can be seen in Fig. 12b.

5) Number of Commands: The number of commands issued by the user follows a similar trend to the task completion time. On average they decreased significantly $(p=0.0013)$ by over two-thirds from 18, when using discrete shared control, to only 5, when using proportional shared control.

6) Assistance Factor: Again, as expected, the assistance factor was significantly higher under the proportional shared control paradigm (0.68 as opposed to $0.02, p=0.0058)$, due 


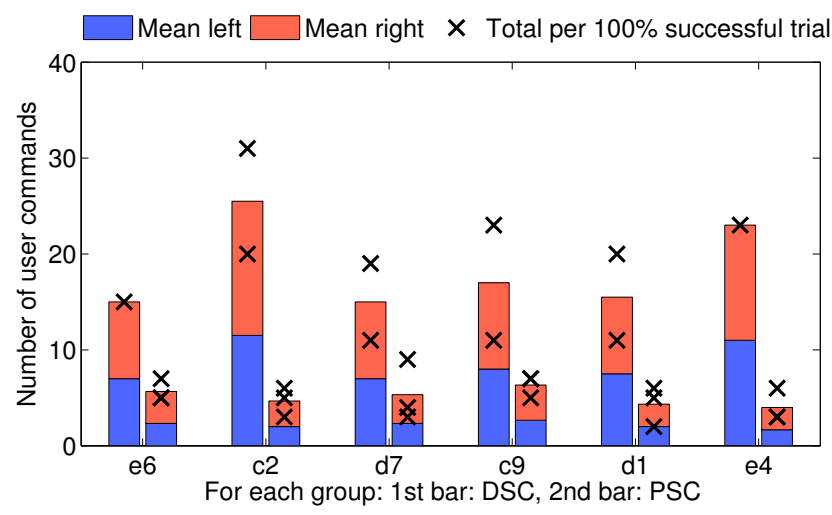

Fig. 13: Number of user commands ([Discrete/Propotional] Shared Control) $(p=0.0013)$.

to the additional proactive turns that were made. This time, in the cluttered environment, it was much easier to see when the proportional shared controller was issuing proactive commands and likewise, when the discrete shared controller was stopping and waiting for the user to make an informed decision (see Fig. 9).

7) Qualitative Analysis: After the experiment, the participants were again asked if they noticed any difference in the wheelchair's behaviour and if so, did they have a preference for one of conditions? In the cluttered environment, every participant responded that they noticed a difference in the behaviour and moreover, all participants preferred to use the proportional shared controller. In this environment with less reflective floors, no false positives were generated by the vision system, so the wheelchair did not stop erroneously.

\section{CONCLUSION}

When operating in a cluttered environment and having to make precise manoeuvres, the proportional shared controller far outperformed the discrete controller, both in terms of system and user metrics. Additionally, in this scenario, the participants unanimously preferred to use the proportional controller to complete the task. However, in the open-plan, spacious environment, there was no statistically significant difference in performance between the two control paradigms. Moreover, in this case, the participants themselves perceived little or no difference between the two shared control strategies. In both environments, participants seemed able to quickly form a mental model of the dynamic behaviour of the proportional shared controller, which was perhaps aided by the visual feedback of the obstacles that were perceived by the wheelchair. Therefore, in this study, it seems that the benefits brought by using the proactive proportional shared controller, instead of the reactive discrete shared controller, outweigh any costs.

\section{ACKNOWLEDGMENT}

The authors would like to thank the volunteers who participated in the experiments. This work is supported by the
European ICT Project TOBI (FP7-224631) and the Swiss National Science Foundation through the National Centre of Competence in Research Robotics. This paper only reflects the authors' views and funding agencies are not liable for any use that may be made of the information contained herein.

\section{REFERENCES}

[1] L. Tonin, R. Leeb, M. Tavella, S. Perdikis, and J. del R. Millán, "The role of shared-control in BCI-based telepresence," in Proceedings of 2010 IEEE International Conference on Systems, Man and Cybernetics, 2010.

[2] T. Carlson and Y. Demiris, "Increasing robotic wheelchair safety with collaborative control: Evidence from secondary task experiments," in Proceedings of the IEEE International Conference on Robotics and Automation (ICRA), Anchorage, AK, May 2010, pp. 5582-5587.

[3] K. M. Tsui, D. J. Feil-Seifer, M. J. Matarić, and H. A. Yanco, "Performance evaluation methods for assistive robotic technology," in Performance Evaluation and Benchmarking of Intelligent Systems, R. Madhavan, E. Tunstel, and E. Messina, Eds. Springer, 2009, pp. 41-66.

[4] D. Norman, The Design of Everyday Things. Doubleday Business, 2002.

[5] T. Carlson, "Collaborative control mechanisms for an intelligent robotic wheelchair," Ph.D. dissertation, Imperial College London, 2010.

[6] H. Yanco, "Wheelesley, a robotic wheelchair system: Indoor navigation and user interface," in Assistive Technology and Artificial Intelligence, ser. Lecture Notes in Computer Science, V. Mittal, H. Yanco, J. Aronis, and R. Simspon, Eds. Springer, 1998, pp. 256-268.

[7] T. Taha, J. Miro, and G. Dissanayake, "POMDP-based long-term user intention prediction for wheelchair navigation," in Proceedings of IEEE International Conference on Robotics and Automation (ICRA), Pasadena, LA, May 2008, pp. 3920-3925.

[8] D. Abbink and M. Mulder, Neuromuscular Analysis as a Guideline in designing Shared Control. IN-TECH, 2010, ch. 27, pp. 499-516.

[9] P. Nisbet, "Who's intelligent? Wheelchair, driver or both?" in Proceedings IEEE International Conference on Control Applications, Glasgow, Scotland, U.K., September 2002.

[10] M. Batavia, A. I. Batavia, and R. Friedman, "Changing chairs: anticipating problems in prescribing wheelchairs," Disability \& Rehabilitation, vol. 23, no. 12, pp. 539-548, 2001.

[11] L. Fehr, W. E. Langbein, and S. B. Skaar, "Adequacy of power wheelchair control interfaces for persons with severe disabilities: a clinical survey," Journal of Rehabilitation Research and Development, vol. 37, no. 3, pp. 353-600, 2000 .

[12] S. Levine, D. Bell, L. Jaros, R. Simpson, Y. Koren, and J. Borenstein, "The navchair assistive wheelchair navigation system," IEEE Transactions on Rehabilitation Engineering, vol. 7, pp. 443-451, 1999.

[13] F. Galán, M. Nuttin, E. Lew, P. W. Ferrez, G. Vanacker, J. Philips, and J. D. R. Millán, "A brain-actuated wheelchair: Asynchronous and non-invasive brain-computer interfaces for continuous control of robots." Clinical Neurophysiology, vol. 119, no. 9, pp. 2159-2169, Sep 2008.

[14] J. Millán, R. Rupp, G. Müller-Putz, R. Murray-Smith, C. Giugliemma, M. Tangermann, C. Vidaurre, F. Cincotti, A. Kübler, R. Leeb, C. Neuper, K. Müller, and D. Mattia, "Combining brain-computer interfaces and assistive technologies: State-of-the-art and challenges," Frontiers in Neuroscience, vol. 4, p. 161, 2010.

[15] J. d. R. Millán and J. Carmena, "Invasive or noninvasive: Understanding brain-machine interface technology [conversations in bme]," Engineering in Medicine and Biology Magazine, IEEE, vol. 29, pp. 16-22, 2010.

[16] T. Carlson and Y. Demiris, "Human-wheelchair collaboration through prediction of intention and adaptive assistance," in Proceedings of the IEEE International Conference on Robotics and Automation (ICRA), Pasadena, CA, 2008, pp. 3926-3931.

[17] T. Carlson, G. Monnard, and J. Millán, "Vision-based shared control for a BCI wheelchair," in Tools for Brain Computer Interaction Workshop (TOBI Workshop 2), Rome, Italy, December 2010.

[18] J. Borenstein and Y. Koren, "The vector field histogram - fast obstacle avoidance for mobile robots," IEEE Transactions on Robotics and Automation, vol. 7, no. 3, pp. 278-288, 1991. 